\title{
Features of sonographic parameters of liver in practically healthy men of different somatotypes, in the Podilski region of Ukraine
}

\author{
Igor Gunas $^{1}$, Marina Melnik ${ }^{2}$, Olexandr Majewskyi², Yuriy ShevchuK ${ }^{2}$
}

${ }^{1}$ International Academy of Integrative Anthropology, Vinnytsia, Ukraine

${ }^{2}$ Vinnitsa National Medical University named after M.I. Pirogov, Vinnytsia, Ukraine

\section{ARTICLE INFO \\ Received 12 May 2017 \\ Accepted 12 June 2017}

\section{Keywords:}

liver,

ultrasound,

healthy men,

constitutional differences.

\begin{abstract}
The analysis of liver sonographic parameters of healthy men of the Podilski region of Ukraine who are of different somatotypes has revealed significant differences only in the thickness of the right lobe of the liver on inhalation, as well as in the width of the caudate lobe of the liver and the densitometry of the left lobe of the liver parenchyma on exhalation and during inhalation. No significant differences or trends between healthy men of different somatotypes were seen in the rest of the sonographic parameters of liver (oblique vertical size of the right lobe of the liver on exhalation and on inhalation, the thickness of the right lobe of the liver on exhalation, the cranio-caudal size of the left lobe of the liver on exhalation and inhalation, the thickness of the left lobe of the liver on exhalation and in inhalation, as well as the length of the caudate particle of the liver and densitometry of right lobe of liver parenchyma on exhalation and inhalation).
\end{abstract}

\section{INTRODUCTION}

One of the more informative, safe and affordable methods for diagnosing liver disease is the ultrasound test. This shows changes in the work of this organ and allows the diagnostician to analyze situations that deviate from the norm, as well as to assess the structure, size and manifestations of liver disease in the early stages of their development [19, 21]. It should be noted that diseases such as cirrhosis, portal hypertension, benign and malignant tumors require precise dynamic morphometric control during conservative surgery or chemo-radiation treatment [15].

In undertaking an ultrasound examination, the diagnostician measures the size of the liver and compares this with the standard parameters $[5,21]$. The performance and results of such research can differ to the norm, depending on the person age, sex and body mass [1,16,20,21]. Attention is drawn to the fact that the anatomical variability indices of ultrasound investigations of the liver are also determined by somatotype, and, accordingly, without the constitutional characteristics of the surveyed, variability can be seen as abnormal increase or decrease of organ [8].Population studies of constitutional characteristics of liver in practically healthy individuals significantly complements the

\footnotetext{
* Corresponding author

e-mail: freekozak1@gmail.com
}

existing morphological data about regularities of growth and development of the inhabitants of a certain region at various stages of ontogeny. Moreover, these can serve as a scientific basis in the development of regional biomedical health promotion programs [13].

The aim of this study was to determine the features of sonographic parameters of the liver in healthy men of different somatotypes, from the Podilski region of Ukraine.

\section{MATERIALS AND METHODS}

The study was undertaken at the Scientific and Research Center of Vinnitsa National Medical University named after M.I. Pirogov (Ukraine), wherein, after an initial survey, 602 practically healthy men aged from 22 to 35 years were recruited. They were representatives of Slavic ethnic groups in the third generation who are residing in the Podilski region of Ukraine. After the secondary screening estimation of state of health, carried out using a special questionnaire and by psychophysiological and psychogigienic testing, as well as through careful clinical and laboratory studies, 93 healthy men served as the basis for the conducted anthropometric survey.

The Podilski region is located on the Ukrainian crystalline shield of granitic rock, and the population is exposed to 
the influence of local manifestations of natural radioactivity brought about by elevated concentrations of uranium in the crystalline rocks. The structure of Podilski region includes three regions (Ternopil, Khmelnytsky and Vinnitsa) with a total area of 60.9 thousand square $\mathrm{km}$ and a population of 4.6 million people evenly distributed rurally and urbanely. The peculiarity of the area is that it contains the highest proportion $(90 \%)$ of the Ukrainian population. This regional population belongs to the Danubian anthropological type and displays Europeoid features. According to F. Vovk [22], the Podilski Ukrainian, as an anthropological type, only slightly differs from the general type. Hence, research results of anthropometric characteristics can be applied to the population of other regions of Ukraine.

Echometric indicators of the liver were measured utilizing the ultrasound diagnostic system "CAPASEE" SSA-220A (Toshiba, Japan), and a convex detector with a working frequency of $3.75 \mathrm{MHz}$, under conventional methods [18]. We defined the oblique vertical size of the right lobe of the liver on exhalation and inhalation; the thickness of the right lobe of liver during inhalation and on exhalation; the cranio-caudal size of the left lobe of the liver during inhalation and on exhalation; the thickness of the left lobe of the liver during inhalation and on exhalation; the length of the caudate part of the liver; the width of the caudate part of the liver; the densitometry of the right lobe of the liver parenchyma during inhalation and on exhalation; as well as the densitometry of the left lobe of the liver parenchyma during inhalation and on exhalation.

Anthropometric examination was conducted in accordance with the scheme of V.V. Bunak, but as modified by P.P. Shaparenko [17]. To evaluate the somatotype, we used the mathematical scheme of J.L. Carter and B.H. Heath [7].

Statistical analysis of the results was conducted by way of the package "STATISTICA 6.1” (license № AXXR910 A374605FA), employing nonparametric methods of evaluation of the result.

The study was approved by the Bioethics Committee Vinnitsa National Medical University named after M.I. Pirogov (protocol number 7 of 08.06.2015), and the study corresponds to the bioethical and moral-legal requirements of the Helsinki Declaration, the European Convention on Human Rights and Biomedicine (1977), the relevant provisions of the WHO and the laws of Ukraine.

\section{RESULTS}

The results of the study of sonographic parameters of the liver in healthy men of different somatotypes are given in Table 1.

In this comparison, we identified the following significant differences: Value 1: thickness of the right lobe of the liver during inhalation, of men of the endo-mesomorphic somatotype. This was significantly greater $(\mathrm{p}<0.01)$ when compared to that of men of the ecto-mesomorphic somatotype; Value 2: width of the caudate lobe of the liver, of men of the endo-mesomorphic somatotype. This was significantly less $(p<0.05)$ when compared to that of men of the mesomorphic somatotype; Value 3: The densitometry of the left lobe of the liver parenchyma on exhalation, in men of the mesomorphic somatotype. This was significantly less $(\mathrm{p}<$ 0.05), when compared with that of men of the ecto-mesomorphic somatotype; Value 4: The densitometry value of the parenchyma of the left lobe of the liver on inhalation, of men of the mesomorphic somatotype. This was significantly less $(p<0,05-0,01)$ when compared with that of men of the ecto-mesomorphic and endo-mesomorphic somatotypes.

Thus, among several number parameters of liver ultrasound, only the thickness of the right lobe of the liver during inhalation, the width of the caudate lobe of the liver, and densitometry indicators of the left lobe of the liver parenchyma on exhalation and on inhalation revealed somatotype differences.

Table 1. Percentile scope of sonographic parameters of liver in healthy men with different somatotypes, from the Podilski region of Ukraine

\begin{tabular}{|l|c|c|c|}
\hline Sonographic sizes & $\begin{array}{c}\text { Mesomorph } \\
(\mathrm{n}=42)\end{array}$ & $\begin{array}{c}\text { Ecto-mesomorph } \\
(\mathrm{n}=12)\end{array}$ & $\begin{array}{c}\text { Endo-mesomorph } \\
(\mathrm{n}=21)\end{array}$ \\
\hline OVSRLE $(\mathrm{mm})$ & $142.0-155.0$ & $139.5-149.0$ & $144.0-160.0$ \\
\hline OVSRLB $(\mathrm{mm})$ & $109.0-131.0$ & $110.5-123.0$ & $113.5-128.5$ \\
\hline TRLE $(\mathrm{mm})$ & $134.0-145.0$ & $130.5-140.5$ & $130.0-146.0$ \\
\hline TRLB $(\mathrm{mm})$ & $111.0-128.0$ & $109.5-117.0$ & $117.0-129.0$ \\
\hline CCSLLE $(\mathrm{mm})$ & $97.5-108.0$ & $93.5-113.0$ & $96.4-107.0$ \\
\hline CCSLLB $(\mathrm{mm})$ & $100.0-113.0$ & $100.5-114.0$ & $100.0-107.0$ \\
\hline TLLE $(\mathrm{mm})$ & $59.5-67.0$ & $57.0-65.3$ & $59.0-71.0$ \\
\hline TLLB $(\mathrm{mm})$ & $60.0-68.0$ & $57.5-69.0$ & $61.3-73.6$ \\
\hline LCP $(\mathrm{mm})$ & $40.5-51.0$ & $45.0-51.5$ & $36.4-45.0$ \\
\hline WCP $(\mathrm{mm})$ & $18.4-24.0$ & $17.8-21.3$ & $13.9-19.7$ \\
\hline DRLPE $(\mathrm{dB})$ & $8.6-11.9$ & $8.8-12.1$ & $8.0-12.0$ \\
\hline DRLPB $(\mathrm{dB})$ & $8.6-11.1$ & $9.7-13.1$ & $9.2-13.4$ \\
\hline DLLPE $(\mathrm{dB})$ & $9.1-11.8$ & $10.4-13.2$ & $10.1-12.6$ \\
\hline DLLPB $(\mathrm{dB})$ & $8.7-11.4$ & $10.0-13.1$ & $9.7-13.5$ \\
\hline
\end{tabular}

Footnote: OVSRLE and OVSRLB - oblique vertical size of the right lobe of the liver on inhalation and on exhalation; TRLE and TRLB - the thickness of the right lobe of the liver on inhalation and on exhalation; CCSLLE and CCSLLB cranio-caudal size of the left lobe of the liver on inhalation and on exhalation; TLE and TLB - the thickness of the left lobe of the liver on inhation and on TLLE and TLLB - the thickness of the left lobe of the liver on inhalation and on exhalation; LCP - the length part the caudate of the liver; WCP - the width of
the caudate part of the liver; DRLPE and DRLPB - densitometry of the right lobe of the liver parenchyma on inhalation and on exhalation; DLLPE and DLLPB - densitometry of the left lobe of the liver parenchyma on inhalation and on exhalation

\section{DISCUSSION}

Current literature mostly lists averages data about the size of the liver, pancreas and gall bladder, and this is not accompanied by additional information on the type of constitution [5,20]. What is more, previous studies often are only of the children-adolescent and youth ages [3,4,21]. It is known that changes can occur in the constitutive parts of the liver in disease situations. For example, with cirrhosis, a size increase can be seen in the caudate that is concurrent with a reduction in size of the left lobe and right lobe [12]. In the liver, the right lobe is the most functionally active, thus, at pathology, its size (especially thickness) can vary considerably [15]. Still, a number of authors also point to the autonomy of the caudate lobe of the liver, which has been confirmed by changes in certain pathological conditions. For example, with Budd-Chiari disease, the blood flow from the liver is sent through the caudate lobe, which then significantly increases in size $[12,15]$. What is more, 
the densitometry of the liver parenchyma is important in the diagnosis of acute and chronic hepatitis, cirrhosis, metabolic disorders, calcifications and others diseases that, in addition to determining the type and severity, need long-term control of «positive response» to therapy [19].

In a number of studies, anatomical variability in the liver was found via ultrasound of individual of different ages that had been determined by somatotype and by anthropometric parameters such as height and weight $[1,12]$. Indeed, according to A.V. Kondrashev et al. [11] and O.V. Chaplygin [8], in individuals at time of puberty, except for the diameter of the portal vein, significant differences can be seen in liver ultrasounds of individuals of different somatotypes, the value of each tending to increase in the number from microsomal to macrosomal type of constitution. In contrast to the above, there are works in which variations in liver sizes, in infants, children and adolescents, is influenced by age and gender differences more than by constitution [16, 20,21]. Such differences can be significant, in contrast to that of adults, wherein, constitution brings about greater differences $[16,20,21]$. The above is easily explained, because, in teenagers and youth, there is a natural increased growth of soma and internal organs [14], and this is most evident in the liver [10,21].

When analyzing the data derived through ultrasound examination of the pancreas and gallbladder of individuals of different somatotypes, several researchers have noted that the average value of all the studied parameters in subjects belonging to different somatotypes were significantly different from the average "good" values of these indices, hence, there is a somatotypical predetermination of the studied sizes $[3,9]$.

According to data of N.V. Belik [2], in practically healthy boys of the ectomorphic somatotype, the gallbladder size is the smallest, while in mesomorphs, it is the largest. Our results are, however, closer to the data obtained by A.V. Kondrashev et al. [11], wherein all the studied indicators of gallbladder also significantly differ among representatives of different somatotypes and tend to increase in the number of micro-macrosomia. This should be considered when interpreting the data of ultrasound examination of the abdomen. Thus, in deciding the question, is there an increase or decrease of the investigated organs that is related to somatotype, diagnosticians at first must pay attention not to the average standards of their size, but at the features of their configuration and the dimensional characteristics in the representatives of various constitutional types. These results bring us closer to understanding the concept of «population norm» for the above parameters and enable diagnosis pathologies of these organs, which are accompanied by changes in their size, even at the preclinical stage of the disease.

\section{CONCLUSIONS}

In almost healthy men of the endo-mesomorphic somatotype, significantly greater $(\mathrm{p}<0.01)$ thicknesses of the right lobe of the liver are evident during inhalation, when compared to that of the ecto-mesomorphic somatotype, while significant lower values $(p<0.05)$ were seen in the width of the caudate lobe of the liver, when compared with that of the mesomorphic somatotype. Moreover, in men of the mesomorphic somatotype, a significant lower $(\mathrm{p}<0.05-0.01)$ value of densitometry was noted in the left lobe of the liver parenchyma on exhalation, when compared with that of the ecto-mesomorphic somatotype, while a significantly less value of densitometry of the left lobe of the liver parenchyma was seen during inhalation, when compared with that of the ecto-mesomorphic and endo-mesomorphic somatotypes.

\section{REFERENCES}

1. Ayede A. et al.: Normal ultrasonographic dimensions of the liver in neonates in South-West Nigeria. West Afr. J. Med., 33, 183, 2014.

2. Belik N.V. et al.: Age, gender and somatotypological features size and shape of the gallbladder in healthy urban adolescents of Podillia. Biomedical and Biosocial Anthropology, 4, 39, 2005.

3. Belik N.V.: Features morphometric characteristics of pancreas in urban adolescents based on age, gender and somatotype. Bulletin of Vinnitsa National Medical University, 9, 205, 2005.

4. Belik N.V.: Ultrasound parameters of liver and spleen in urban adolescents with different somatotype. Bulletin of Vinnitsa State Medical University, 7, 3, 2003.

5. Capaccioli L. et al.: The anteroposterior diameter of the head and the body of the pancreas in healthy Italian children. Ital. J. Anat. Embryol., 98, 3, 1993.

6. Capaccioli L. et al.: Ultrasonographic study on the growth and dimensions of healthy children and adults organs. Ital. J. Anat. Embryol., 105, 1, 2000.

7. Carter J.E.L. (2002). The Heath-Carter anthropometric somatotype. Instruction Manual. Surrey, Canada: TeP and Rosscraft; p.154.

8. Chaplygina E.V (2009). Somatotypological laws of anatomical variability of the liver and gall bladder in people youthful and first period of mature age. Volgograd; p.197.

9. Chaplygina H.V. et al.: Some sonographic parameters of abdominal organs in healthy children and adolescents with known somatometric parameters. Materials of the $8^{\text {th }}$ Congress of the Asian Federation of Societies for ultrasound in Medicine and Biology, Thailand Bangkok, 2007.

10. Gould S.I.: Allometry and size in ontogeny and phylogeny. Biol. Revs., 41, 587, 1966.

11. Kondrashev A.V. et al.: Age, sexual and type features of morphofunctional characteristics of organs of the hepatobiliary system in persons of adolescence according to ultrasound data. Biomedical and Biosocial Anthropology, 9, 2007.

12. Kratzer W., Fritz V., Mason R.A.: Factors affecting liver size: a sonographic survey of 2080 subjects. J. Ultrasound Med., 22, 1155, 2003.

13. Nykytyuk B.A., Moroz V.M., Nykytyuk D.B. (1998). Theory and practice of integrative anthropology. Essays. Kiev-Vinnitsa: Zdorovia; p.301.

14. Omelka F., Bystry J.: Somatotypes and body height dimensions of children aged from 9 to 18. Antropol., 10, 1/2, 1987.

15. Rutkauskas S. et al.: Clinical and anatomical basis for the classification of the structural parts of liver. Medicina, 42, 98, 2006.

16. Salome N.E., Anyanwu G.E., Obikili N.E.: Normal Sonographic evaluation of normal liver sizes of school children in south-east Nigeria. West Afr. J. Med., 2, 29, 2014.

17. Shaparenko P.P. (2000). Anthropometry. Vinnitsa; p.71.

18. Tchacarski V. et al. (2015). Atlas of diagnostic ultrasound. First English Edition. Sofia.

19. Tchelepi H. et al.: Sonography of diffuse liver disease. J. Ultrasound Med., 21, 1023, 2002. DOI: 10.7863/jum.2002.21.9.1023.

20. Udoaka A.I., Enyi C., Agi C.E.: Sonological evaluation of the liver, spleen and the kidneys in an adult Southern Nigerian population. Asian Journal of Medical Sciences, 5, 2, 2012.

21. Urata K. et al.: Standard liver volume in adults. Transplant. Proc., 32, 2093, 2000. 
Igor Gunas, Marina Melnik, Olexandr Majewskyi, Yuriy Shevchuk

22. Vovk H.V. (1995). Anthropological features of the Ukrainian people. Studies of Ukrainian Ethnography and anthropology. K: .Art; p.7-38. 